\title{
Newly manufactured Marukome MK-34-1 miso with angiotensin- converting enzyme inhibitory activity and its antihypertensive effects in genetic hypertensive rat models
}

\author{
Hiroe Sakuyama Tomari ${ }^{1}$ Misa Uchikawa ${ }^{1}$ - Aki Yamazaki ${ }^{1}$ - Satomi Hirabayashi ${ }^{1}$ - Shoko Yamakawa ${ }^{1}$. \\ Manabu Kitagawa ${ }^{2} \cdot$ Minami Yamada $^{2} \cdot$ Seiki Itou $^{2} \cdot$ Tetsuro Yamamoto $^{3} \cdot$ Yoshio Uehara $^{1}$
}

Received: 10 July 2018 / Revised: 20 November 2018 / Accepted: 22 November 2018 / Published online: 10 January 2019

(c) The Author(s) 2019. This article is published with open access

\begin{abstract}
We newly manufactured miso rich in angiotensin-converting enzyme (ACE) inhibitory activity (Marukome MK-34-1, shinki miso) and investigated its antihypertensive properties in rat models of genetic hypertension. ACE inhibitory activity was tenfold higher in shinki miso than in commercially available Marukome Nenrin miso (nenrin miso). The inhibitory activity of shinki miso was confined to $<3 \mathrm{kDa}$ fractions and was detected in several fractions with high polarity by $\mathrm{C}_{18}$ highperformance liquid chromatography. Systolic blood pressure (SBP) increased age-dependently in stroke-prone spontaneously hypertensive rats (SHRSP/Izm) given a $0.6 \%(\mathrm{w} / \mathrm{v}) \mathrm{NaCl}$ solution (salt solution group) that matched the salt content of the miso solutions. This SBP increase was attenuated in both the 5\% nenrin and 5\% shinki miso solution groups compared to the salt solution group. The reduction in SBP was greater in rats fed shinki than in rats fed nenrin miso. Similarly, in a salt-induced hypertension model with Dahl rats, the 5\% nenrin miso solution attenuated the rising SBP observed in the salt solution group. Moreover, combining 5\% nenrin miso with 5\% shinki miso (2:1, v/v) (awase miso group) significantly decreased the SBP per gram salt intake by $8 \%$ compared with the nenrin miso treatment. However, there were no differences in urinary $\mathrm{Na}$ excretion between the nenrin and awase miso groups. In conclusion, we produced a new miso with potent ACE inhibitory activity that reduced spontaneous and salt-induced hypertension. These results suggest that salt sensitivity is decreased by the addition of shinki miso to nenrin miso.
\end{abstract}

Keywords Miso $\cdot$ ACE $\cdot$ SHRSP $\cdot$ Dahl rats $\cdot$ Hypertension

\section{Introduction}

Miso is a traditional Japanese food whose consumption dates to $700 \mathrm{AD}$. It is usually produced from soybeans with malted rice and salt. One serving of miso soup contains $1-2$ $\mathrm{g}$ of salt. Therefore, traditional miso soup has long been assumed to be the reason for the high incidence of saltsensitive hypertension, which can potentially cause cerebral stroke, in Japan.

Yoshio Uehara

yoshiodr@nn.iij4u.or.jp

1 Division of Clinical Nutrition, Faculty of Home Economics, Kyoritsu Women's University, Tokyo, Japan

2 Marukome Co. Ltd., Nagano, Japan

3 TTC Co. Ltd., Tokyo, Japan
We recently demonstrated that miso soup attenuates saltinduced hypertension in Dahl rats prone to salt-induced hypertension (Dahl $\mathrm{S}$ rats) [1, 2]. This was attributed to natriuresis or vasodilation induced by miso soup ingredients. Intraperitoneal or subcutaneous administration of a very small amount of miso extract decreased the blood pressure (BP) in Dahl S rats [3], an effect that has also been investigated in clinical settings. However, in a human intervention trial, two daily servings of miso soup ( $3.8 \mathrm{~g}$ salt/day) for 3 months did not affect the systolic blood pressure (SBP) or diastolic blood pressure (DBP) of subjects with normotension or stage 1 hypertension [4]. Moreover, a 5-year crosssectional observation found no association between the frequency of miso soup intake and the SBP and DBP of subjects with normal or high normal BP [5].

Some studies have suggested that miso contains compounds that attenuate the BP response to high salt intake [15]. Although these hypotensive factors remain to be 
identified, it can be supposed that increasing hypotensive factor content would promote miso consumption as a health food. In fact, enzymatically digested soybeans were found to reduce $\mathrm{BP}$, and nicotianamine extracted from soybeans inhibited angiotensin-converting enzyme (ACE) activity [6, 7]. Our preliminary experiments showed that miso crude extract inhibits ACE activity in vitro by $10-20 \%$ (unpublished data).

We speculated that miso with potent ACE inhibitory activity attenuates hypertension to a greater extent than regular commercially available miso, particularly hypertension associated with enhancement of the renin-angiotensin system (RAS). To test this hypothesis, we produced a novel type of miso (Marukome MK-34-1) with potent ACE inhibitory activity and examined its hypotensive effects in rat models of spontaneous and salt-induced hypertension.

Long-term diuresis is associated with Na imbalance and decreased body fluid space. Such disruption leads to enhanced RAS activity with attenuation of BP reduction. Here, to examine whether shinki miso works as an ACE inhibitor, we tested the possible synergistic effects of regular miso (Marukome Nenrin) and miso with potent RAS inhibitory activity.

\section{Methods}

\section{Characterization of hypotensive properties of shinki miso in a spontaneous hypertension model, SHRSP/ Izm}

Male stroke-prone spontaneously hypertensive/Izumo rats (SHRSP/Izm) (8 weeks old, $n=30$ ) were purchased from Sankyo Laboratory (Tokyo). The rats were randomly divided into three equal-sized groups (ten rats in each group) that were given (1) 5\% (w/v) Marukome MK-34-1 miso (shinki miso, Nagano) solution with potent ACE inhibitory activity $(0.6 \% \mathrm{NaCl}, \mathrm{w} / \mathrm{v})$ (shinki group); (2) $5 \%$ (w/v) commercially available Marukome Nenrin miso solution $(0.6 \% \mathrm{NaCl}, \mathrm{w} / \mathrm{v})$ (nenrin group); or (3) $0.6 \% \mathrm{NaCl}(\mathrm{w} / \mathrm{v})$ solution (salt solution group). The rats were all fed regular chow $(0.6 \% \mathrm{NaCl}, \mathrm{w} / \mathrm{w})$ (Oriental Kobo MF, Tokyo) for 4 weeks. Miso or salt solutions and chow were freely available throughout the experiment.

\section{Combined effects of shinki miso and nenrin miso on salt-induced hypertension in Dahl rats}

Male Dahl salt-sensitive (Dahl S/Iwai) rats (4 weeks old, $n$ $=24$ ) were purchased from Sankyo Laboratory and were fed regular chow $(0.6 \% \mathrm{NaCl}, \mathrm{w} / \mathrm{w})$ chow and given $1 \%$ $\mathrm{NaCl}(\mathrm{w} / \mathrm{v})$ solution as drinking water. After 3 weeks, the rats were randomly divided into three equal-sized groups (eight rats in each group) that were given (1) $0.6 \% \mathrm{NaCl}(\mathrm{w} /$ v) solution (salt solution group); (2) a $2: 1(\mathrm{v} / \mathrm{v})$ combination of $5 \%$ nenrin miso $(0.6 \% \mathrm{NaCl}, \mathrm{w} / \mathrm{v})$ and $5 \%$ shinki miso with potent ACE inhibitory activity $(0.6 \% \mathrm{NaCl}$, w/v) solutions (awase group); or (3) 5\% nenrin miso solution $(0.6 \% \mathrm{NaCl}, \mathrm{w} / \mathrm{v})$ (nenrin group). The rats were fed regular chow $(0.6 \% \mathrm{NaCl}, \mathrm{w} / \mathrm{w})$ (Oriental Kobo MF) for 4 weeks. Miso or salt solutions and chow were freely available throughout the experiment. Food consumption was measured every 2-3 days.

\section{Preparation of miso solution}

We utilized 5\% (w/v) nenrin miso solutions because we had reported that conventional miso solutions with concentrations $>5 \%$ (w/v) attenuate salt-induced hypertension in Dahl $\mathrm{S}$ rats $[1,2]$. The salt concentration in the $5 \%(\mathrm{w} / \mathrm{v})$ nenrin solution was determined using a Na Meter Model TS-999i (Toko Chemical Laboratories Co. Ltd., Tokyo). Then, shinki miso was manufactured to make the salt concentration equal to that in nenrin miso.

Miso dissolved in distilled water (5\%, w/v) was homogenized using an ultrasonicator. Then, the homogenate was autoclaved at $120^{\circ} \mathrm{C}$ for $1 \mathrm{~h}$. Insoluble residues were removed using a cotton filter, and the soluble fraction was used as the miso solution. The solution was stored at $4{ }^{\circ} \mathrm{C}$ until use. The preparation was repeated every $2-3$ days.

\section{BP measurement}

SBP was measured weekly by the tail-cuff method as previously described but with a modified detection system (Model KN-210-1; Natsume Seisakujo, Tokyo) [8]. The same investigator measured the SBP of all animals in a quiet, warm room between 10:00 and 14:00 $\mathrm{h}$.

\section{Sample collection}

At the end of the experiment, the rats were placed in a metabolic cage to collect 24-h urine samples [1, 2]. The volume of salt and miso solutions consumed by the rats was measured every 1-3 days. Each animal was anesthetized with pentobarbital sodium (i.p., $75 \mathrm{mg} / \mathrm{kg}$ body weight), and then the kidney, heart, and aortic wall along with the blood were collected. The aortic wall samples $(1 \mathrm{~cm}$ segments) were obtained from the descending thoracic aorta just distal to the aortic arch and then weighed. Urine and blood samples were stored at $-80{ }^{\circ} \mathrm{C}$ until assayed.

\section{Characterization of shinki miso}

A batch of regular commercially available nenrin miso was supplied by Marukome Co. Ltd., and stored at $-20^{\circ} \mathrm{C}$ until 
Table 1 Characterization of Marukome shinki miso

\begin{tabular}{lll}
\hline & Shinki & Nenrin \\
\hline Materials & $\begin{array}{l}\text { Soybeans, rice, salt, } \\
\text { ethanol, free of yeast }\end{array}$ & $\begin{array}{l}\text { Soybeans, rice, salt, } \\
\text { ethanol, yeast }\end{array}$ \\
$\begin{array}{l}\text { Percentage of malted } \\
\text { rice }^{\mathrm{a}}\end{array}$ & $50 \%$ & $70 \%$ \\
$\begin{array}{l}\text { Species of Koji starter } \\
\text { Salt content }\end{array}$ & Not open & Aspergillus oryzae \\
$\begin{array}{l}\text { Fermentation term } \\
\text { ACE inhibitory }\end{array}$ & 5 days & $12.1 \%$ \\
activity ${ }^{\text {(IC }}$ (I0) & $0.23 \mathrm{mg} / \mathrm{mL}$ & 46 days \\
\hline
\end{tabular}

Shinki, Marukome shinki miso rich in ACE inhibitory activity; nenrin, Marukome Nenrin miso

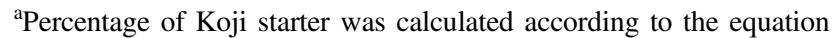
$100 \times$ malted rice $(\mathrm{g}) /$ soybean $(\mathrm{g})$

${ }^{\mathrm{b}} \mathrm{ACE}$ inhibitory activity was determined according to the method previously reported [9-11]

use to prevent further fermentation. The new shinki miso with potent ACE inhibitory activity was originally produced by Marukome [9]. The main characteristics are listed in Table 1. The new shinki miso was prepared in the same manner as nenrin miso using soybeans, malted rice, and salt but with a much shorter fermentation period (5 vs 46 days). Additionally, different species of Koji starter were used for the two types of miso, the details of which cannot be disclosed due to the patent policy.

\section{Evaluation of ACE inhibition}

ACE activity was determined according to an established method [9-11]. Briefly, miso extract was mixed with standard ACE and $25 \mathrm{mM}$ hippuryl-histidyl-leucine substrate in HEPES buffer ( $\mathrm{pH}$ 8.3). The cleaved histidyl-leucine emitted a fluorescence signal (excitation/emission, 340/ $485 \mathrm{~nm}$ ) in the presence of $0.2 \% o$-phthalaldehyde in methanol. The inhibitory activity was calculated with the following equation: activity $=100 \times(1-\mathrm{ACE}$ activity with miso extract/ACE activity without miso). The half-maximal inhibitory concentrations $\left(\mathrm{IC}_{50} \mathrm{~s}\right)$ were 0.23 and $2.5 \mathrm{mg} / \mathrm{mL}$ for new and regular miso, respectively.

\section{Measurement of urinary and blood parameters}

Electrolytes in blood and urine were measured using an autoanalyzer (Model 736; Hitachi Co., Tokyo). Urinary protein concentrations were measured using a protein assay kit (Bio-Rad, Hercules), while urinary catecholamine was measured by high-performance liquid chromatography (HPLC; SRL Laboratories, Tokyo). Plasma and urine osmolality was measured by the standard molecular freezing point depression method (SRL Laboratories).
Measurements were performed by investigators who were blinded to the group assignments.

\section{Evaluation of oxidative stress in the kidney}

Since the RAS is closely associated with upregulation of oxidative stress, we investigated the relationship between oxidative stress and renal function in rats given miso solution. To assess oxidative stress in the kidney, we estimated lipid peroxide levels in kidney homogenates based on malondialdehyde (MDA) production [12, 13]. Briefly, kidney tissue was homogenized in ice-cold Dulbecco's balanced phosphate-buffered saline using an ultrasonicator and centrifuged. A portion of the supernatant was incubated at $37^{\circ} \mathrm{C}$ for $30 \mathrm{~min}$. The generated lipid peroxides were converted to MDA with trichloroacetate. The product was coupled with thiobarbiturate, and the density was determined at a wavelength of $532 \mathrm{~nm}$. Protein levels in the supernatant were assayed using a protein assay kit (Bio$\mathrm{Rad})$.

\section{Analysis of miso extract by $\mathrm{C}_{18}$ reversed-phase HPLC}

Miso extract was analyzed by reversed-phase HPLC (COSMOSIL 5C18-AR-II; Naka-Arai Techs, Tokyo) with a $\mathrm{C}_{18}$ column (inner diameter, $6.0 \times 150 \mathrm{~mm}$ ). Briefly, the sample was loaded onto the column and eluted at $0.8 \mathrm{~mL} /$ min and $40{ }^{\circ} \mathrm{C}$ with a $0.1 \%$ trifluoroacetate solution and a 0-80\% acetonitrile gradient for $50 \mathrm{~min}$. The fractionated effluent was evaporated, and the residue was dissolved in phosphate-buffered solution to determine ACE inhibitory activity.

Moreover, we characterized the ACE inhibitory activity in fractions of shinki miso extracts with molecular weights $<3,3-0,10-30$, and $>30 \mathrm{kDa}$ produced with membrane sieves (adjusted to $100 \mathrm{mg}$ extract $/ \mathrm{mL}$ ) (Merck Millipore Corporation, Darmstadt).

\section{Statistical analysis}

All statistical analyses were performed using STATISTICA software (StatSoft, Tulsa). The values are expressed as the mean \pm SD. Differences were assessed by one-way analysis of variance (ANOVA) followed by Fisher's least significant difference post hoc test (LSD test), repeated measures ANOVA, or nonparametric Mann-Whitney $U$ tests. $P$ values $<0.05$ were considered statistically significant.

\section{Guidelines for handling rats in experiments}

The study protocol conformed to the institutional guidelines for experimental animal handling and was approved by the Animal Care Committee of the Kyoritsu Women's 
University (\#16002 and \#17002). The experiments were conducted in accordance with the guidelines of the National Institutes of Health (Bethesda, MD, USA).

\section{Results}

\section{ACE inhibitory activity in the new shinki miso}

The $\mathrm{IC}_{50}$ of ACE inhibition by shinki miso crude extracts was one-tenth that of nenrin miso crude extracts (Table 1). We determined the ACE inhibitory activity in fractions of shinki miso extracts with molecular weights of $<3,3-10$, $10-30$, and $>30 \mathrm{kDa}$ (Fig. 1). For shinki miso, inhibitory activity was confined to the $<3 \mathrm{kDa}$ fraction, whereas for nenrin miso, inhibitory activity was observed in three fractions, although it was mostly in the $<3 \mathrm{kDa}$ fraction. We therefore further analyzed this fraction by $\mathrm{C}_{18}$ reversedphase HPLC and found that the inhibitory activity was present in higher-polarity fractions of shinki miso extract but was limited to fraction 5 of nenrin miso (Fig. 2). In the shinki miso, we found several dipeptides with ACE inhibitory activity in HPLC-MS/NMR (mass-spectrophotometry/nuclear magnetic resonance) analysis (unpublished data). We have not determined a single substance responsible for the ACE inhibitory activity of shinki miso. Several substances may contribute to the apparent ACE inhibitory activity of shinki miso.

\section{Characterization of hypotensive properties of shinki miso in a spontaneous hypertension model, SHRSP/ Izm}

\section{Miso consumption}

The body weight of rats increased in an age-dependent manner in all experimental groups (Fig. 3a). There were no differences in body weight among the three groups during the experimental period. The cumulative amounts of miso or salt solution consumed are shown in Fig. 3b. Consumption was higher in the nenrin miso group than in the salt solution group but was lower in rats fed shinki than in rats fed nenrin miso $(P<0.001)$. As each solution contained $0.6 \% \mathrm{NaCl}$ (w/ $\mathrm{v})$, the cumulative amount of salt obtained from drinking the solution was estimated to be $9.63 \pm 2.19 \mathrm{~g}$ for the salt solution group, $15.31 \pm 2.41 \mathrm{~g}$ for the nenrin miso group $(P<$ 0.05 vs salt solution group), and $12.84 \pm 2.75 \mathrm{~g}$ for the shinki miso group ( $P=0.057$ vs salt solution group).

\section{Effects of shinki miso on systolic blood pressure in SHRSP/Izm}

The organ weights are presented in Table 2. There were no differences in heart and kidney weights among the three

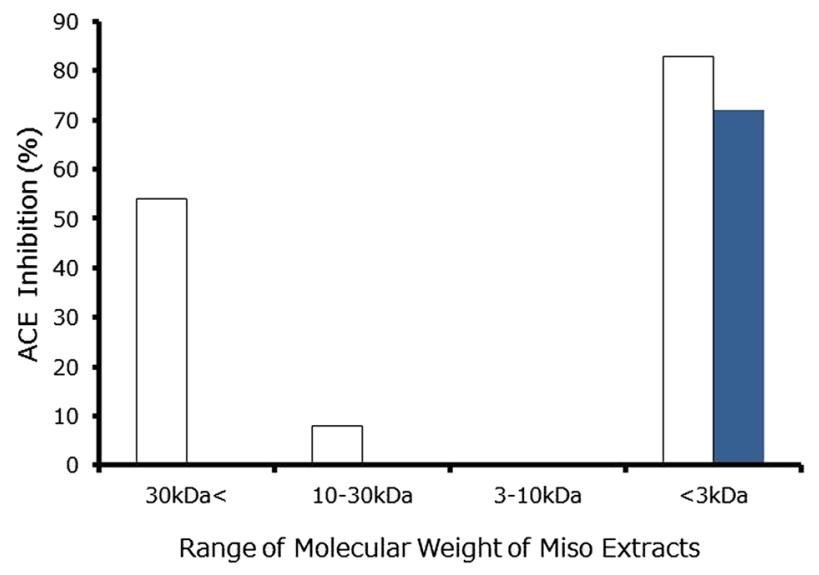

Fig. 1 ACE inhibitory activity of shinki miso. Miso extracts were separated according to molecular weights using membrane sieves as described in the text. In this assay system, the concentrations of both miso extracts to be analyzed were well above the $\mathrm{IC}_{50}(100 \mathrm{mg} / \mathrm{mL})$. The left open column represents the nenrin miso, and the right solid column represents the shinki miso rich in ACE inhibitory activity

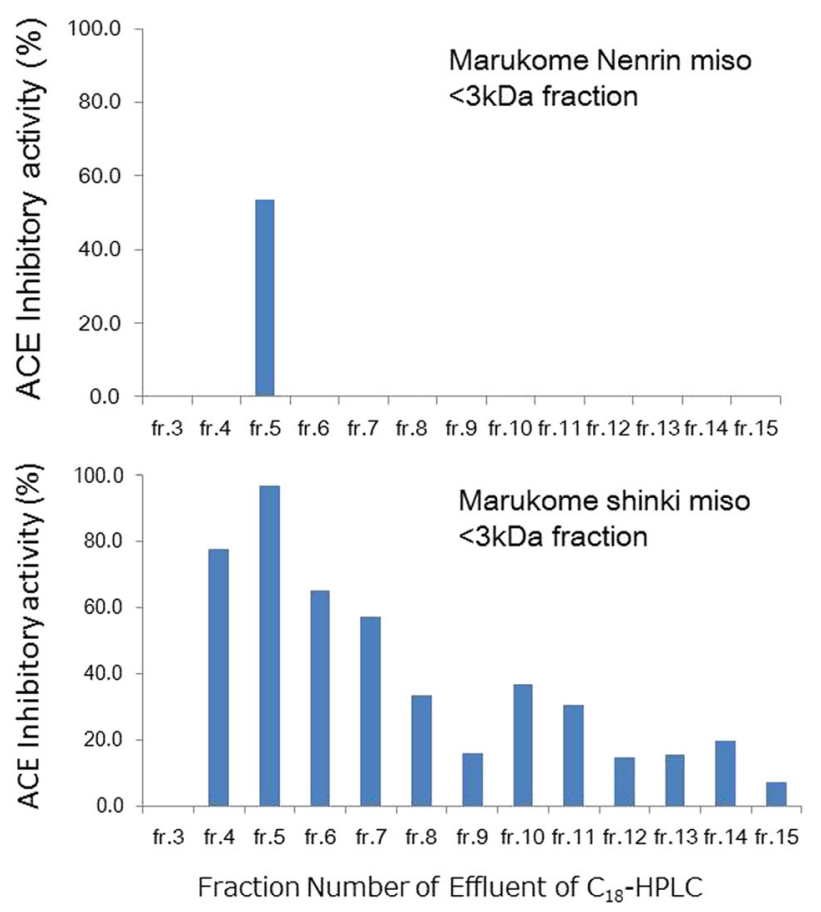

Fig. 2 ACE inhibitory activity in fractionated effluents as determined by $\mathrm{C}_{18}$ reversed-phase HPLC. The upper graph represents $<3 \mathrm{kDa}$ nenrin miso extracts, and the lower graph represents $<3 \mathrm{kDa}$ extracts of shinki miso. The miso extracts $(10 \mu \mathrm{L})$ were analyzed using $\mathrm{C}_{18}$ reversed-phase HPLC as described in the text

experimental groups. In the nenrin miso group, the aortic wall weight was unchanged when compared to that in the salt solution group. In contrast, in the shinki miso group, the aortic wall weight was $8.7 \%$ lower than that in the salt solution group $(P<0.05)$.

SBP increased age-dependently in the three experimental groups (Fig. 4). Even though rats fed miso had a higher salt 
(a)

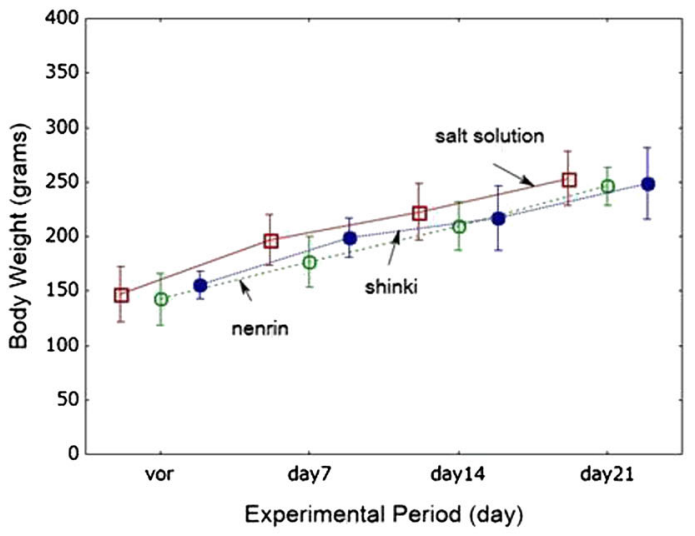

Fig. 3 Body weight changes and cumulative amounts of solution consumption in the experimental groups. The body weight changes are shown in graph (a). The cumulative amounts of consumed miso and salt solutions are shown in graph (b). Open squares, salt solution group; open circles, nenrin miso group; closed circles, shinki miso group. The values in the graphs represent the means $\pm \mathrm{SD}(n=10$ in each group). Group differences in graph (a) were analyzed using oneway analysis of variance (ANOVA) followed by Fisher's least

Table 2 Organ weights in experimental groups

\begin{tabular}{llcl}
\hline Group & Heart & Kidney & Aorta \\
\hline (Units) & $(\mathrm{g} / 100 \mathrm{~g} \mathrm{BW})$ & $(\mathrm{g})$ & $\left(\mathrm{mg} / \mathrm{mm}^{2}\right)$ \\
Salt solution & $0.440 \pm 0.017$ & $1.262 \pm 0.085$ & $0.149 \pm 0.018$ \\
Nenrin & $0.443 \pm 0.026$ & $1.240 \pm 0.058$ & $0.149 \pm 0.014$ \\
Shinki & $0.449 \pm 0.016$ & $1.272 \pm 0.082$ & $0.136 \pm 0.006^{*}, * *$ \\
\hline
\end{tabular}

Salt solution, group given salt solution; nenrin, Marukome Nenrin miso group; and shinki, Marukome shinki miso with potent ACE inhibitory activity miso group. Values are expressed as means \pm SD $(n$ $=10$ in each group). Differences were assessed by one-way analysis of variance (ANOVA) followed by Fisher's least significant difference post-hoc test (LSD test)

$* P<0.05$ vs salt solution, $* * P<0.05$ vs nenrin

intake than those fed the salt solution, SBP was lower in the miso-fed animals. Moreover, SBP was 3.8\% lower at week 2 and $4.1 \%$ lower at week 3 in rats that consumed shinki than in rats that consumed the nenrin miso solution. The $\mathrm{SBP} /$ cumulative salt intake was $13.37 \pm 2.94 \mathrm{mmHg} / \mathrm{g}$ salt intake for the salt solution group, $7.98 \pm 1.29 \mathrm{mmHg} / \mathrm{g}$ salt intake for the nenrin miso group $(P<0.0001$ vs salt solution group), and $9.14 \pm 2.09 \mathrm{mmHg} / \mathrm{g}$ salt intake for the shinki miso group $(P<0.001$ vs salt solution group). There were no differences in salt sensitivity between the shinki miso and nenrin miso groups.

\section{Effects of shinki miso on renal function in SHRSP//zm}

Blood electrolyte concentrations did not differ among the experimental groups (Table 3). Shinki miso influenced (b)

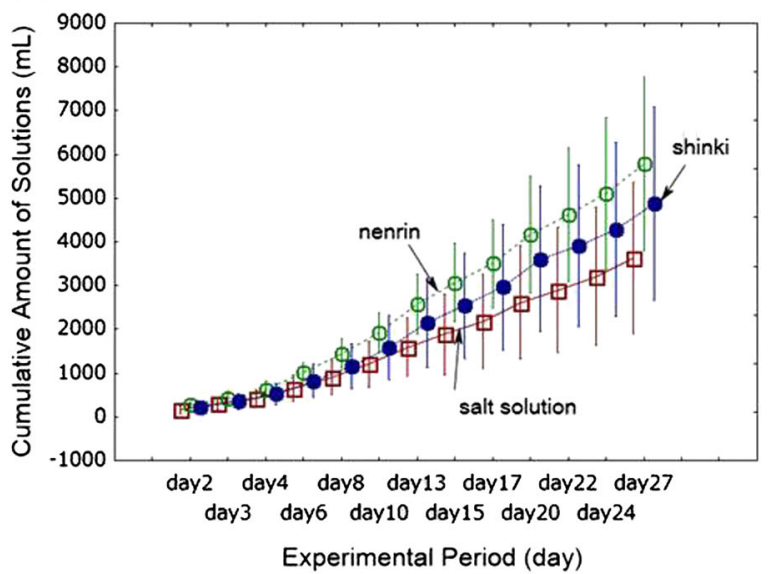

significant difference post hoc test (Fisher LSD test), and those in group (b) were analyzed using repeated measures analysis of variance. There were no group differences in body weights at day 14 and day 21 . The cumulative amounts of miso or salt solution consumed during the experiment differed among the groups $(P<0.001)$. The difference between the salt solution and nenrin miso groups was significant $(P<$ 0.025); however, the consumption of the shinki miso group did not differ from that of the nenrin miso group

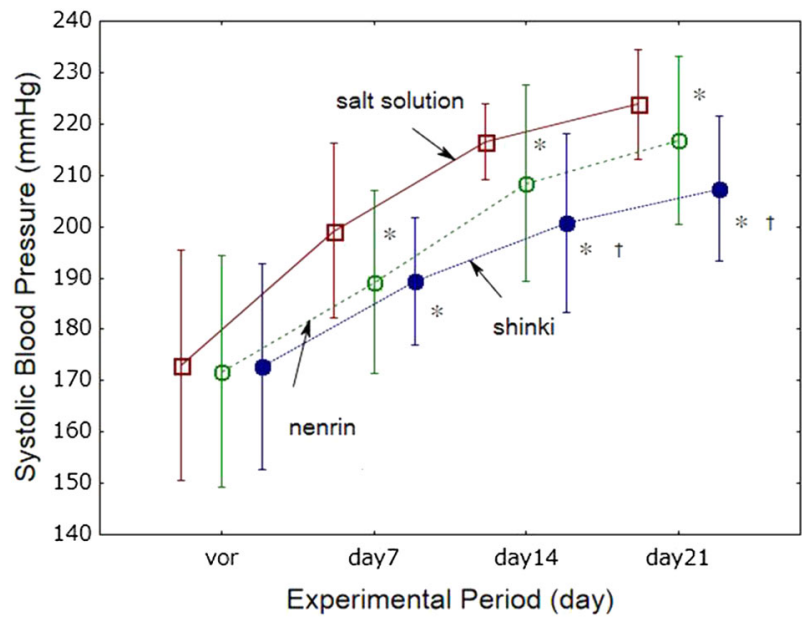

Fig. 4 Effects of shinki miso on systolic blood pressure in SHRSP/ Izm. Open squares, salt solution group; open circles, nenrin miso group; closed circles, shinki miso rich in ACE inhibitory activity group. The values in the graph represent the means $\pm \mathrm{SD}(n=10$ in each group). Group differences were analyzed using repeated measures analysis of variance with Fisher's LSD test $(P<0.001)$. ${ }^{*} P<0.05$ vs salt solution group, $\dagger P<0.05$ vs nenrin miso group

neither urinary nor fractional sodium excretion, whereas urinary sodium excretion was higher in the nenrin miso group than in the salt solution group. Shinki miso did not influence potassium clearance or urinary aldosterone excretion.

Shinki miso influenced neither urinary osmolality clearance nor free water clearance, whereas nenrin miso increased osmolality clearance compared to the salt solution (Table 4). In addition, free-water clearance was decreased in the nenrin 


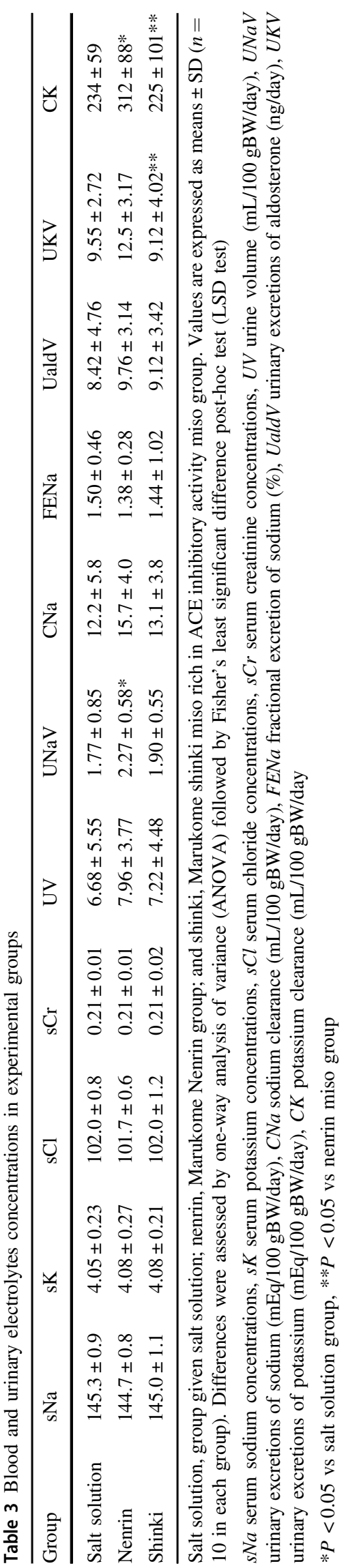

Table 4 The effects of Marukome shinki miso on urinary osmolality and free-water clearance

\begin{tabular}{lllll}
\hline Group & sOsm & Uosm & Cosm & $\mathrm{CH}_{2} \mathrm{O}$ \\
\hline Salt & $299.8 \pm 2.6$ & $1988.7 \pm 789.0$ & $31.9 \pm 11.4$ & $-25.2 \pm 7.1$ \\
solution & & & & \\
Nenrin & $298.4 \pm 2.0$ & $1766.7 \pm 629.8$ & $40.4 \pm 9.5^{*}$ & $-32.4 \pm 6.8^{*}$ \\
Shinki & $298.3 \pm 2.4$ & $1794.2 \pm 886.1$ & $32.2 \pm 7.1$ & $-25.0 \pm 7.5$ \\
\hline
\end{tabular}

Salt solution, group given salt solution; nenrin, Marukome Nenrin miso group; and shinki, Marukome shinki miso rich in ACE inhibitory activity miso group. Values are expressed as means $\pm \mathrm{SD}(n=10$ in each group). Differences were assessed by one-way analysis of variance (ANOVA) followed by Fisher's least significant difference post-hoc test (LSD test)

sOsm plasma osmolarity (mOsm/ $\mathrm{kg} \mathrm{H}_{2} \mathrm{O}$ ), Uosm urinary osmolarity (mOsm/kg $\mathrm{H}_{2} \mathrm{O}$ ), Cosm osmolarity clearance (mL/100 gBW/day), $\mathrm{CH}_{2} \mathrm{O}$ free-water clearance $(\mathrm{mL} / 100 \mathrm{gBW} /$ day $)$

$* P<0.05$ vs salt solution group

miso group, suggesting that rats fed nenrin miso excreted more concentrated urine than rats fed the salt solution.

Urinary creatinine clearance was $33 \%$ higher in the shinki miso group than in the salt solution group; however, the difference was not statistically significant. Creatinine clearance was $36 \%$ higher in rats fed nenrin miso than in rats fed salt solution only (Fig. 5a). There were differences in urinary protein excretion, a target of angiotensin II in the kidney, among the three groups, but the differences were not significant (Fig. 5b).

\section{Shinki miso and oxidative stress in the kidney}

Since angiotensin II levels may decrease due to the ACE inhibitory activity of miso, which could reduce NADPH oxidase activity and oxidative stress, we tested whether the new miso decreased oxidative stress in the kidneys by measuring MDA production [14-16]. Shinki miso reduced MDA formation compared to the salt solution (Fig. 5b). Moreover, MDA formation in the kidney was positively correlated with urinary protein excretion $(r=0.47, P<0.05)$.

\section{Effects of shinki miso on urinary catecholamine excretion}

There were no differences in urinary catecholamine excretion between the shinki miso and salt solution groups (Table 5). However, rats in the nenrin miso group excreted higher levels of urinary noradrenaline and dopamine than those in the salt solution group, although the differences were not significant.

\section{Combined effects of shinki miso and nenrin on salt-induced hypertension in Dahl rats}

SBP increased in a time-dependent manner in Dahl $\mathrm{S}$ rats given the salt solution (Fig. 6). This increase was time- 
(a)

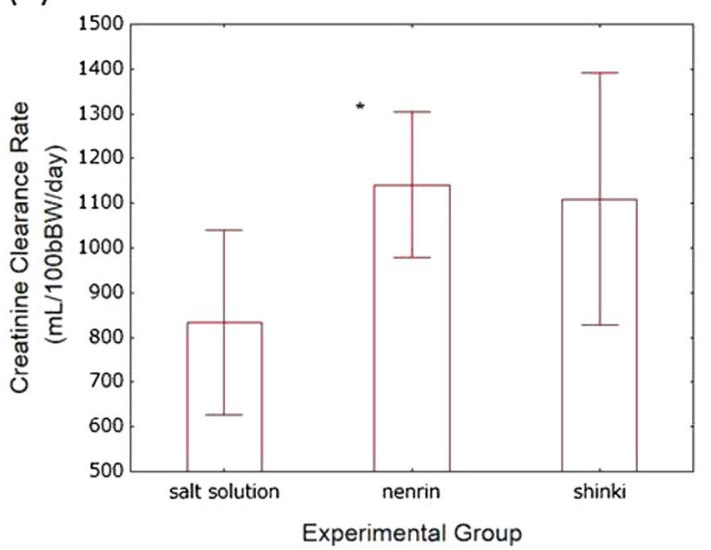

Fig. 5 Effects of shinki miso on renal function in SHRSP/Izm. Creatinine clearance rates are shown in graph (a). The effects of miso on urinary protein excretion and oxygen stress are shown in graph (b). Salt solution, group given salt solution; nenrin, nenrin miso group;

Table 5 Effects of Marukome shinki miso on catecholamine excretions in urine

\begin{tabular}{llll}
\hline Group & UadrV & UnorV & UdopaV \\
\hline Salt solution & $3.75 \pm 2.67$ & $45.1 \pm 48.6$ & $305.1 \pm 259.7$ \\
Nenrin & $4.32 \pm 1.91$ & $58.5 \pm 52.3$ & $446.9 \pm 255.2$ \\
Shinki & $4.56 \pm 2.49$ & $36.9 \pm 15.8$ & $404.1 \pm 235.2$ \\
\hline
\end{tabular}

Salt solution, group given salt solution; nenrin, Marukome Nenrin miso group and; shinki, Marukome shunki miso group. Values are expressed as means $\pm \mathrm{SD}$ ( $n=10$ in each group)

UadrV urinary excretions of adrenaline ( $\mu \mathrm{g} / 100 \mathrm{gBW} /$ day), UnorV urinary excretions of noradrenaline $(\mu \mathrm{g} / 100 \mathrm{gBW} /$ day), UdopaV urinary excretions of dopamine $(\mu \mathrm{g} / 100 \mathrm{gBW} /$ day $)$

dependently attenuated in rats fed nenrin miso, with a statistically significant difference in the nenrin miso group relative to the salt solution group on day 28. Similarly, in rats that consumed a combination of nenrin and shinki miso, SBP was lower on days 14 and 28 than that in the salt solution group.

The cumulative volume of drinking solution consumed increased over time in the three groups (Fig. 7a); however, rats in the miso groups drank more solution than those in the salt solution group, and the amount of solution consumed was higher in the awase group than in the nenrin group. Similarly, food consumption was greater in the miso groups than in the salt solution group (Fig. 7b). However, there was no significant difference in cumulative food consumption between the awase miso and nenrin miso groups.

Based on these data, we estimated the total (cumulative) salt intake during the experiment. The awase miso group consumed more salt $(16.2 \pm 0.5 \mathrm{~g} / 28$ days $)$ than the nenrin $(15.1 \pm 0.3 \mathrm{~g} / 28$ days) and salt solution $(12.4 \pm 1.2 \mathrm{~g} /$ (b)

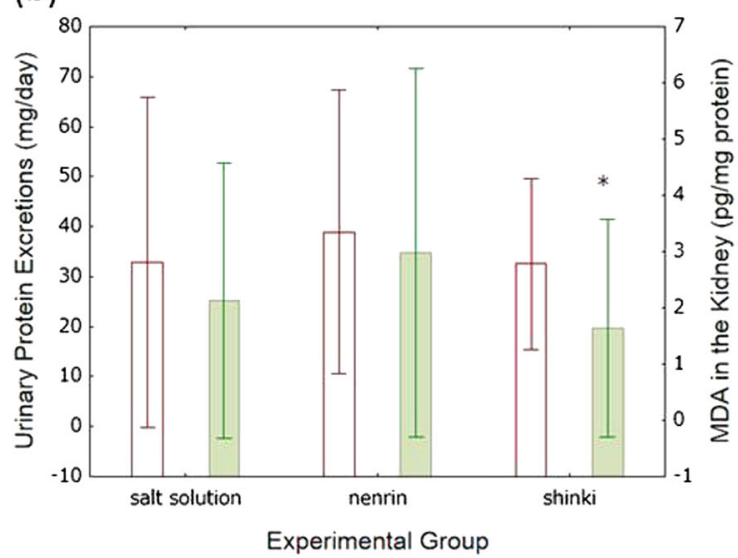

shinki, shinki miso rich in ACE inhibitory activity group. The values in the graph represent the means $\pm \mathrm{SD}$ ( $n=10$ in each group). Differences were assessed with Mann-Whitney $U$ tests. $* P<0.05$ vs salt solution group

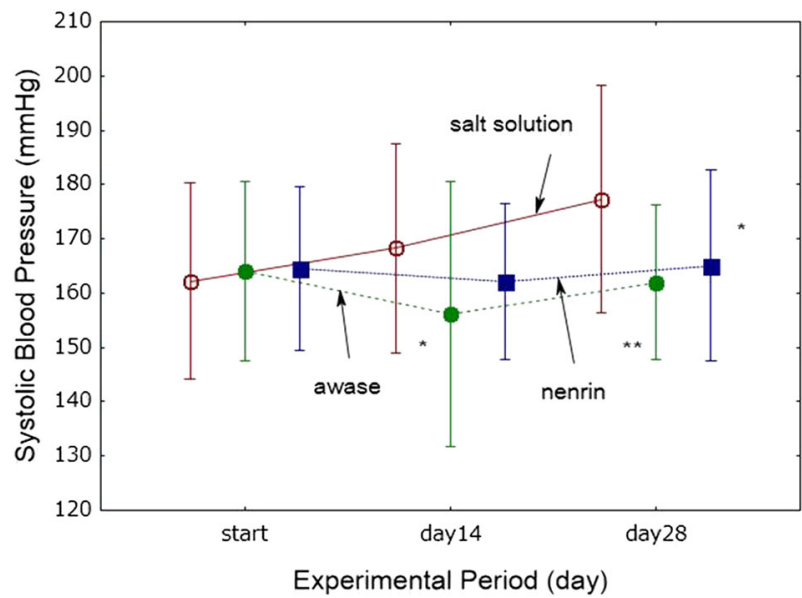

Fig. 6 Combined effects of shinki miso and nenrin on salt-induced hypertension in Dahl rats. Salt solution, group given salt solution; awase, group given a combination of shinki and nenrin miso; nenrin, group given nenrin miso. Differences were analyzed by one-way ANOVA followed by Fisher's LSD test. The values in the graph represent the means $\pm \mathrm{SD}$ ( $n=8$ in each group). $* P<0.05$, $* * P<$ 0.001 vs salt solution group

28 days) groups (Fig. 8a). Since salt intake differed among the experimental groups, we normalized SBP on day 28 to cumulative salt intake (Fig. 8b). SBP per gram of salt intake was significantly lower in the nenrin miso group than in the salt solution group. Moreover, SBP per gram of salt intake was significantly lower by $8 \%$ in rats fed a combination of shinki and nenrin miso (awase miso) than in rats fed nenrin miso.

Urinary $\mathrm{Na}$ excretion as a function of salt intake over 28 days was increased in both miso groups relative to the salt solution group; however, there was no difference between the awase and nenrin miso groups (Fig. 9). 
(a)

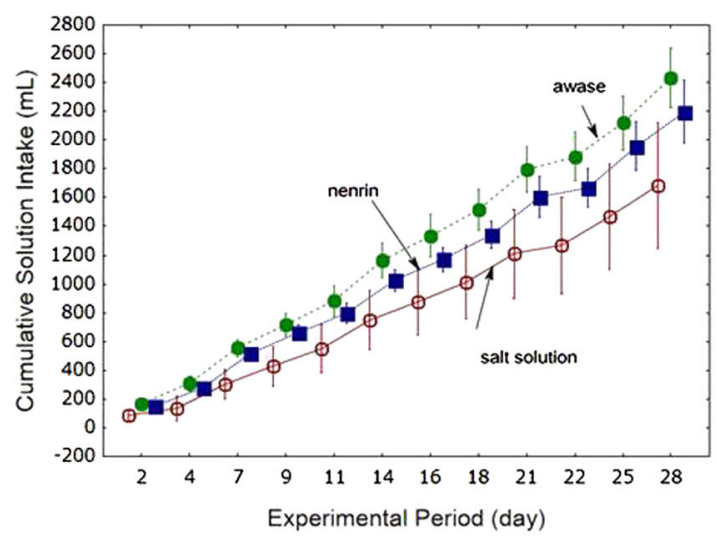

Fig. 7 Cumulative solution intake and food consumption in Dahl saltsensitive rats. The cumulative solution intake during the experiment is shown in graph (a), and food consumption is shown in graph (b). Salt solution, group given salt solution; awase, group given a combination of shinki and nenrin miso; nenrin, group given nenrin miso. The values in the graph represent as the means $\pm \mathrm{SD}$ ( $n=8$ in each group). Differences among groups in the cumulative amount of solutions

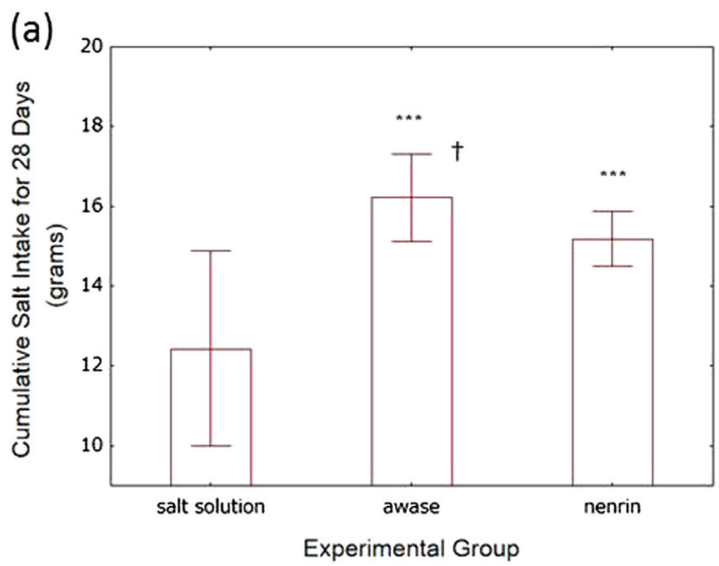

Fig. 8 Cumulative salt intake and SBP on day 28 normalized to cumulative salt intake. Based on the amount of solution intake and food consumption over 28 days, the cumulative salt intake was estimated and is shown in graph (a). SBP values normalized to cumulative salt intake during the experiment are shown in graph (b). Salt solution,

\section{Discussion}

The main objective of this study was to produce new miso with hypotensive effects by modifying the fermentation process. Many studies have reported that there is ACE inhibitory activity in enzyme-hydrolyzed fermented soybean or dipeptides derived from soybean [17-19]. To our knowledge, however, there have been few studies in vivo on the involvement of ACE inhibition in BP reduction by miso. The most important finding of the present study is that we have manufactured miso with potent ACE inhibitory activity and that this miso may work as an ACE inhibitor to attenuate hypertension. This finding indicates that the awase (b)

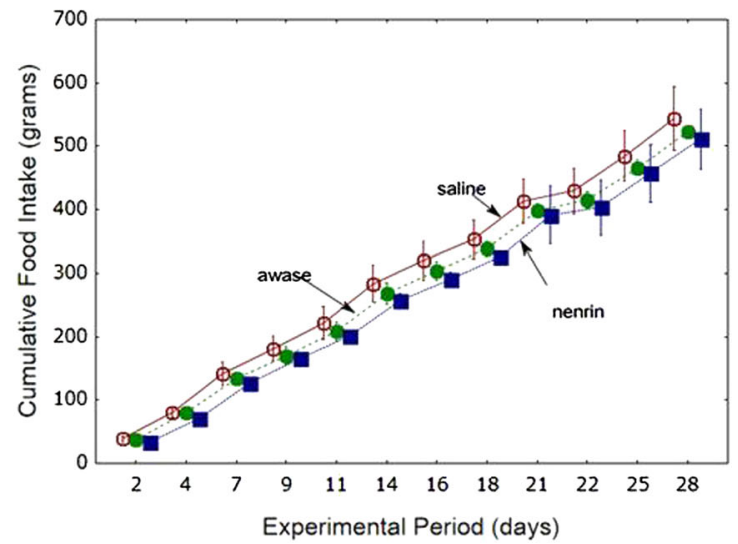

consumed were assessed using repeated measures analysis of variance with Fisher's LSD test $(P<0.0001)$, and the amount of awase miso consumed was higher than that of nenrin miso $(P<0.005)$. Differences in cumulative food consumption were significant among the three groups $(P<0.025)$; however, there was no difference in food consumption between the awase miso and nenrin miso groups

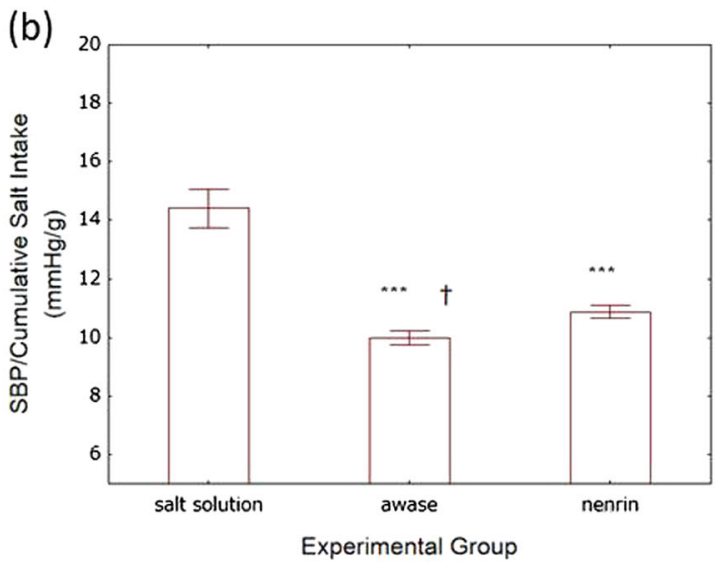

group given salt solution; awase, group given a combination of shinki and nenrin miso; nenrin, group given nenrin miso. The values in the graph represent the means $\pm \mathrm{SD}(n=8$ in each group). Differences were analyzed by one-way ANOVA followed by Fisher's LSD test. $* * * P<0.001$ vs salt solution, $\dagger P<0.05$ vs nenrin

miso technique is a strategy to easily produce tasteful miso with antihypertensive effects. In this sense, this is the first study to report the role of ACE inhibition in miso in antihypertensive effects and the benefits of a combination of conventional miso and miso with ACE inhibitory activity.

In this study, we produced miso that has high ACE inhibitory activity (shinki miso) and investigated its hypotensive properties in SHRSP/Izm, a genetic model of spontaneous hypertension [1, 2, 5-7]. These rats are prone to cerebral stroke and show enhanced activation of the RAS and adrenergic nervous system. The most important finding of the present study was that this newly manufactured miso with potent ACE inhibitory activity attenuated spontaneous 


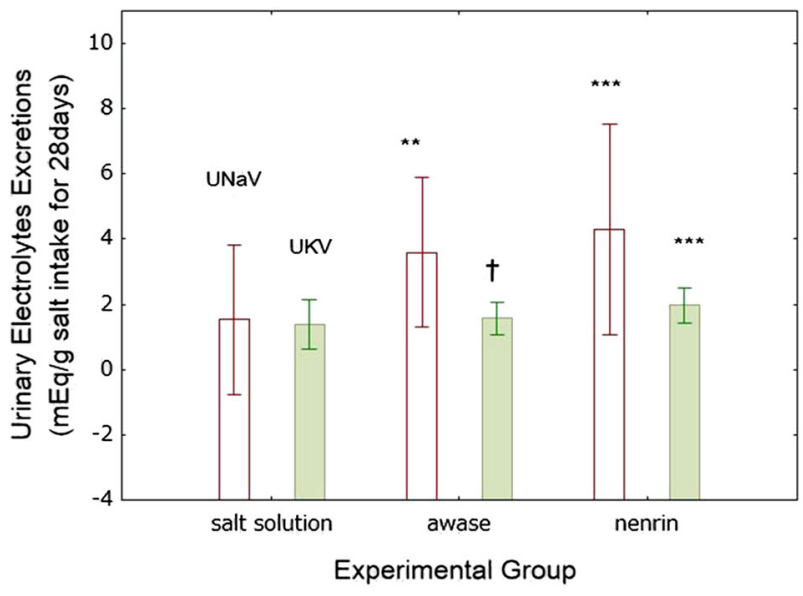

Fig. 9 Urinary excretion of sodium and potassium. Salt solution, group given salt solution; awase, group given a combination of shinki and nenrin miso; nenrin, group given nenrin miso. The values in the graph represent the means $\pm \mathrm{SD}$ ( $n=8$ in each group). Differences were analyzed by one-way ANOVA with Fisher's LSD test. $* * * P<0.001$ vs salt solution, $\dagger P<0.05$ vs nenrin

hypertension in this model. BP decreases were greater in rats fed shinki miso than in rats fed nenrin miso, which is used for daily cooking in Japan. Shinki miso differs from nenrin miso in terms of the Koji starter that is used and the fermentation period. Our results suggest that a slight modification in the manufacturing process, e.g., the species of Koji starter, and potentiation of ACE inhibitory activity contribute to a greater reduction in BP.

We previously demonstrated that long-term intake of traditional miso attenuates salt-induced hypertension through sodium diuresis or vasodilation in Dahl S rats [1, 2]. We also showed that BP reduction in SHRSP/Izm fed nenrin miso was associated with increased urinary sodium excretion. However, in the shinki miso group, there was no increase in urinary sodium excretion, suggesting that the observed reduction in BP was attributable to a mechanism distinct from sodium regulation in the kidneys.

However, to examine the possible natriuretic effects of miso, we assessed urinary $\mathrm{Na}$ excretion in the acute phase and $\mathrm{Na}$ space in a chronic study. We estimated the ratio of body weights to cumulative salt intake. In Dahl rats at day 28 , the ratios were $25.4 \pm 2.3 \mathrm{~g} \mathrm{BW} / \mathrm{g}$ salt intake for the salt solution group, $18.0 \pm 1.1 \mathrm{~g} \mathrm{BW} / \mathrm{g}$ salt intake for the awase miso group $(P<0.05$ vs the nenrin miso group and $P<$ 0.001 vs the salt solution group, Mann-Whitney $U$ test), and $20.1 \pm 1.4 \mathrm{~g} \mathrm{BW} / \mathrm{g}$ salt intake for the nenrin miso group $(P<0.001$ vs the salt solution group, Mann-Whitney $U$ test). In the nenrin and awase miso groups, the ratios were significantly lower than that in the salt solution group. This may be explained by a reduction in body fluid space, because the average body weights differed by only $5 \%$. In particular, body weight did not differ between the awase and nenrin miso groups, so the difference in the ratio may be due to a decrease in body fluid space in the awase miso group. However, our findings do not provide direct evidence regarding body fluid space on a long-term basis.

It is conceivable that natriuresis due to nenrin miso and RAS inhibition by shinki miso synergistically decrease saltinduced hypertension. To test this hypothesis, we examined whether the combination of nenrin and shinki miso (awase miso) affected salt-induced hypertension in Dahl S rats and found that BP reduction in the awase miso group was greater than that in the nenrin miso group when SBP was normalized to the amount of salt intake (Fig. 8b). There were no differences in urinary $\mathrm{Na}$ excretion between the two miso groups, suggesting that the addition of shinki miso enriched in ACE inhibitory activity enhanced the hypotensive effects of nenrin miso not by promoting natriuresis but through mechanisms related to vasodilation $[1,2]$.

ACE inhibition and reduced angiotensin II concentrations in blood are presumed to reduce aldosterone secretion, thereby decreasing sodium reabsorption in aldosteronesensitive distal tubules. However, urinary aldosterone excretion was not diminished by shinki miso, and potassium clearance was unaltered in SHRSP/Izm. The reason for this finding is unclear; however, it is known that suppression of aldosterone by ACE inhibitors can be attenuated even while BP remains controlled [14, 15]. This so-called aldosterone escape may have occurred in the shinki miso group.

In the present study, we did not measure plasma angiotensin concentrations due to the limited amount of blood samples, nor did we determine ACE activity in the SHRSP/ Izm study. In the awase miso study, ACE activity was $12.81 \pm 4.47 \mathrm{IU} / \mathrm{L}$ for the salt solution $(n=4), 16.68 \pm 6.98$ IU/L for nenrin miso $(n=3)$, and $12.80 \pm 4.43 \mathrm{IU} / \mathrm{L}$ for awase miso $(n=5)$. ACE activity tended to be elevated in nenrin miso compared to the salt solution and may have shifted toward that of the salt solution group when nenrin miso was combined with shinki miso. However, the difference was not significant, and the findings are not conclusive because of the limited samples available to determine ACE activity.

Approximately $50-100 \mathrm{~mL}$ of $5 \%$ miso solution $(2.5-5 \mathrm{~g}$ of miso) was consumed by each rat per day. Considering that the average body weight of a rat was $250 \mathrm{~g}$ and that the body fluid accounts for $60 \%$ of body weight in general, the concentration of miso components in the blood was estimated to be $10-20 \mathrm{mg} / \mathrm{g} \mathrm{BW}$ or approximately $16-33 \mathrm{mg} /$ $\mathrm{mL}$ body fluid. This estimate is much higher than the $\mathrm{IC}_{50}$ of shinki miso $(0.23 \mathrm{mg} / \mathrm{mL})$, suggesting that shinki miso consumption was enough to suppress the RAS in SHRSP/ Izm and Dahl $S$ rats. Data on the bioavailability of active substances were not determined except for salt. We have found that salt in miso is absorbed almost completely through the gastrointestinal tract. However, we could not 
determine the bioavailability of the ACE inhibitory substance(s), and estimation remains to be performed.

Major antihypertensive effects may be caused by decreased angiotensin II and subsequent vasodilation or facilitation of $\mathrm{Na}$ excretion in the kidney. However, it is technically difficult to directly prove the relationship between plasma angiotensin II reduction and these events. Since renal oxygen stress and aortic wall thickness are mediated by angiotensin II upregulation, however, decreased renal oxygen stress and aortic wall thickness probably reflect decreases in plasma angiotensin II, consistent with the findings of a previous study on an ACE inhibitor, alacepril [20]. Moreover, we demonstrated in a Dahl S rat study that salt sensitivity was slightly but significantly decreased through $\mathrm{Na}$ diuresis when shinki with ACE inhibitory activity was combined with nenrin miso. Taken together, the results suggest that it is conceivable that the RAS was downregulated by shinki miso under the experimental conditions in the present study.

We demonstrated that oxidative stress in the kidneys of SHRSP/Izm was lower in rats fed shinki miso that had high ACE inhibitory activity than in those that consumed a nenrin miso solution. Angiotensin II generates oxygen radicals through activation of NADPH oxidase [16, 21, 22]. The decreased oxidative stress in the shinki miso group could reflect inhibition of the RAS in the kidneys [23]. We also demonstrated that the aortic wall weight of SHRSP/Izm decreased by $8.7 \%$ in the shinki miso group compared to the salt solution group but was unaltered in the regular miso group. This could be explained, in part, by regional RAS inhibition by shinki miso. Moreover, ACE inhibition slows bradykinin degradation and enhances generation of prostacyclin and nitric oxide [23-25], which dilate vessels and reduce vascular smooth muscle cell proliferation [26-28]. These mechanisms could contribute to remodeling of vascular walls in the shinki miso group.

In our previous studies, rats were fed miso that was fermented for 3 or more months [1-3]; urinary sodium excretion and dopamine secretion were higher in these rats than in those that consumed salt solutions. In the present study, we compared the effects of miso fermented for 46 and 5 days. Our results suggest that the fermentation period is critical for sodium handling in the kidneys. However, the precise manner in which the fermentation period provides health benefits remains to be investigated.

\section{Perspectives}

In summary, we showed that a new miso with high ACE inhibitory activity (Marukome shinki miso) has antihypertensive effects in spontaneous and salt-induced hypertension. The antihypertensive mechanisms of shinki miso differed from those of regular commercially available miso (Marukome Nenrin) and potentiated the antihypertensive properties of the regular miso in salt-induced hypertension. New types of miso enriched in specific ingredients with health benefits to humans could have broad applications in the food culture of Japan.

Acknowledgements All authors except for Manabu Kitagawa, Minami Yamada, and Seiki Itou are responsible for the content and writing of this paper. The coauthors Manabu Kitagawa, Minami Yamada, and Seiki Itou contributed to manufacturing nenrin and shinki miso; they did not participate in or perform any part of the SHRSP/Izm and Dahl $S$ rat studies, analyze the data, or participate in preparing the manuscript.

Funding This study was supported in part by a grant from the Kyoritsu Women's University (2016) and Marukome Co., Ltd., Nagano, Japan (2016 and 2017).

\section{Compliance with ethical standards}

Conflict of interest The authors declare that they have no conflict of interest.

Publisher's note: Springer Nature remains neutral with regard to jurisdictional claims in published maps and institutional affiliations.

Open Access This article is licensed under a Creative Commons Attribution 4.0 International License, which permits use, sharing, adaptation, distribution and reproduction in any medium or format, as long as you give appropriate credit to the original author(s) and the source, provide a link to the Creative Commons license, and indicate if changes were made. The images or other third party material in this article are included in the article's Creative Commons license, unless indicated otherwise in a credit line to the material. If material is not included in the article's Creative Commons license and your intended use is not permitted by statutory regulation or exceeds the permitted use, you will need to obtain permission directly from the copyright holder. To view a copy of this license, visit http://creativecommons. org/licenses/by/4.0/.

\section{References}

1. Yoshinaga M, Toda N, Tamura Y, Terakado S, Ueno M, Otsuka $\mathrm{K}$, et al. Japanese traditional Miso soup attenuates salt-induced hypertension and its organ damage in Dahl salt-sensitive rats. Nutrition. 2012;28:924-31.

2. Du D, Yoshinaga M, Sonoda M, Kawakubo K, Uehara Y. Blood pressure reduction by Japanese traditional Miso is associated with increased diuresis and natriuresis through dopamine system in Dahl salt-sensitive rats. Clin Exp Hypertens. 2014;6:359-66.

3. Shimizu N, Du D, Sakuyama H, Ito Y, Sonoda M, Kawakubo K, et al. Continuous subcutaneous administration of Miso extracts attenuates salt-induced hypertension in Dahl salt-sensitive rats. Food Nutr Sci. 2015;6:693-702.

4. Kitagawa M, Itoh K, Yamada M, Koike S, Yamamoto T, Uehara Y. Long term intake of miso soup unaffected blood pressure in subjects with normal or stage I hypertension-double blind comparative intervention trial. Jpn Pharmacol Ther. 2016;44:1601-12. (abstract in English)

5. Sakuyama H, Tachizaki N, Nanmo A, Yamada K, Uehara Y. The influence of intake of daily Miso soup on blood pressure in 
humans-The results of 5-year observational study. J Jpn Soc Med Use Funct Foods. 2017;10:1-8. (abstract in English).

6. Nevala R, Vaskonen T, Vehniainen J, Korpela R, Vapaatalo H. Soy based diet attenuates the development of hypertension when compared to casein based diet in spontaneously hypertensive rat. Life Sci. 2000;66:115-24.

7. Takenaka T, Murayama T, Takenaka Y. Isolation of nicotianamine from soybean broth and changes in its content in the soybean steaming process. Nippon Shokuhin Kagaku Kogaku Kaishi. 2009;56:6-13. (abstract in English)

8. Friedman M, Freed SC. Microphonic manometer for indirect determination of systolic blood pressure in the rat. Proc Soc Exp Biol Med. 1949;70:670-2.

9. Kitagawa M, Tada K, Yamada M, Haruno R, Higashi T, Koike S. Purification and characterization of angiotensin I converting enzyme inhibitor from short-term fermented Miso. The 68th Annual Meeting of the Society of Biotechnology of Japan. Toyama, Japan. 2016.

10. Vrielink A1, Obel-Jorgensen A, Codding PW. Hippuryl-L-histidyl-L-leucine, a substrate for angiotensin converting enzyme. Acta Crystallogr C. 1996;52(Pt 5):1300-2.

11. Schwager SL, Carmona AK, Sturrock ED. A high-throughput fluorimetric assay for angiotensin I-converting enzyme. Nat Protoc. 2006;1:1961-4.

12. Uehara Y, Kawabata Y, Shirahase H, Wada K, Hashizume Y, Morishita S, et al. Radical scavenging properties of indapamide and renal protection in Dahl salt-sensitive rats. Hyperten Res. 1992;15:17-26.

13. Uehara $\mathrm{Y}$, Hirawa $\mathrm{N}$, Takeda $\mathrm{T}$, Numabe A, Kawabata $\mathrm{Y}$, Nagoshi $\mathrm{H}$, et al. Possible linkage between injury and cardiac remodeling in Dahl salt-sensitive rats treated with a calcium channel antagonist, benidipine. Hypertens Res. 1995;18:245-53.

14. Jorde UP, Vittorio T, Katz SD, Colombo PC, Latif F, Le Jemtel TH. Elevated plasma aldosterone levels despite complete inhibition of the vascular angiotensin-converting enzyme in chronic heart failure. Circulation. 2002;106:1055-7. https://doi.org/ 10.1161/01.CIR.0000030935.89559.04

15. Bomback AS, Klemmer PJ. The incidence and implications of aldosterone breakthrough. Nat Clin Pract Nephrol. 2007;3: 486-92. https://doi.org/10.1038/ncpneph0575

16. Zafari M, Ushio-Fukai M, Akers M, Yin Q, Shah A, Harrison DG, et al. Role of NADH/NADPH oxidase-derived $\mathrm{H}_{2} \mathrm{O}_{2}$ in angiotensin II-induced vascular hypertrophy. Hypertension. 1998;32: 488-95.

17. Wang Z, Cui Y, Liu P, Zhao Y, Wang L, Liu Y, et al. Small peptides isolated from enzymatic hydrolyzate of fermented soybean meal promote endothelium-independent vasorelaxation and ACE inhibition. J Agric Food Chem. 2017;65:10844-50.

18. Nakahara T, Sugimoto K, Sano A, Yamaguchi H, Katayama H, Uchida R. Antihypertensive mechanism of a peptide-enriched soy sauce-like seasoning: the active constituents and its suppressive effect on renin-angiotensin-aldosterone system. J Food Sci. 2011;76:H201-6.

19. Inoue K, Gotou T, Kitajima H, Mizuno S, Nakazawa T, Yamamoto N. Release of antihypertensive peptides in miso paste during its fermentation, by the addition of casein. J Biosci Bioeng. 2009;108:111-5.

20. Laursen JB, Rajagopalan S, Galis Z, Tarpey M, Freeman BA, Harrison DG. Role of superoxide in angiotensin II-induced but not catecholamine-induced hypertension. Circulation. 1997;95: 588-93.

21. Griendling KK1, Minieri CA, Ollerenshaw JD, Alexander RW. Angiotensin II stimulates NADH and NADPH oxidase activity in cultured vascular smooth muscle cells. Circ Res. 1994;74:1141-8.

22. Hirawa N, Uehara Y, Kawabata Y, Nagata T, Ohshima N, Ono H, et al. Mechanistic analysis of renal protection by angiotensinconverting enzyme inhibitor in Dahl salt-sensitive rats. J Hypertens. 1994; 12:909-8.

23. Bader M. Tissue renin-angiotensin-aldosterone systems: targets for pharmacological therapy. Annu Rev Pharmacol Toxicol. 2010;50:439-65.

24. Owens AP, Subramanian, Moorleghen JJ, Guo Z, McNamara CA, Cassis LA, et al. Angiotensin II induces a region-specific hyperplasia of the ascending aorta through regulation of inhibitor of differentiation 3. Circ Res. 2010;106:611-9.

25. Hirawa N, Uehara Y, Kawabata Y, Ohshima N, Ono H, Nagata T, et al. Subpressodose of angiotensin II increases susceptibility of the haemodynamic injury of blood pressure in Dahl salt-sensitive rats. J Hypertens. 1995;13:81-90.

26. Uehara Y, Takada S, Hirawa N, Kawabata Y, Nagata T, Numabe $\mathrm{A}$, et al. De novo synthesis of phospholipase A2 and prostacyclin production by proliferating rat smooth muscle cells. Prostaglandins. 1993;46:331-46.

27. Uehara Y, Numabe A, Kawabata Y, Takada S, Hirawa N, Nagata $\mathrm{T}$, et al. Inhibition of protein synthesis and antiproliferating effect by angiotensin converting enzyme inhibitor in rat vascular smooth muscle cells. J Hypertens. 1993;11:1073-81.

28. Somoza B, González C, Cachofeiro V, Lahera V, FernándezAlfonso MS. Chronic 1-arginine treatment reduces vascular smooth muscle cell hypertrophy through cell cycle modifications in spontaneously hypertensive rats. J Hypertens. 2004;22: $751-8$. 